\title{
Differential Synapse Formation and Neurite Outgrowth at Two Branches of the Metacerebral Cell of Aplysia in Dissociated Cell Culture $^{1}$
}

\author{
SAMUEL SCHACHER
}

Center for Neurobiology and Behavior, College of Physicians and Surgeons, Columbia University and The New York State Psychiatric institute, New York, New York 10032

\begin{abstract}
The metacerebral cell (MCC) of Aplysia californica was isolated with its bifurcate axon from the cerebral ganglion and maintained in vitro under three conditions: (a) with no targets, (b) with identified buccal ganglion neurons B1 or B2 placed near the stump of the large diameter cerebral-buccal connective (CBC) branch, and (c) with B1 or B2 placed near the stump of the small diameter posterior lip nerve (PLN) branch. After 5 days in culture, the two branches differed significantly in the formation of chemical connections and in the extent of neurite outgrowth. Chemical connections characteristic of MCC-B1(B2) connections in vivo were observed in more than $90 \%$ of the cultures in which the buccal neuron was contacted by neurites emerging from the CBC branch, but in only $20 \%$ of the cultures in which the buccal neuron was contacted by neurites extending from the PLN branch. Neurite outgrowth from the CBC stump was always greater than growth from the PLN and was not affected significantly by the presence of a buccal neuron target at either branch. In contrast, neurite outgrowth from the PLN decreased significantly when the target was contacted by neurites from the CBC branch. These results suggest that two branches of a single neuron can differ in their capacities to form chemical connections. In addition, the two branches show differential growth as a result of target interaction at one of the branches. This simple in vitro system may therefore be useful in exploring the ways in which individual neurons control neurite extension from different branches as they seek to form chemical connections with their targets.
\end{abstract}

Neurite outgrowth during development or regeneration is regulated by a number of factors. For example, soluble factors or specific substrate cues may trigger neurite extension and guide axons to their appropriate targets (Letourneau, 1975; Levi-Montalcini, 1976; Varon and Bunge, 1978; Ebendal et al., 1980; Barker et al., 1982; Campenot, 1982; Bentley and Caudy, 1983; Raper et al., 1983). Some neurons, however, extend processes and form connections

Received August 13, 1984; Revised January 2, 1985; Accepted January 29, 1985

\footnotetext{
1 This work was supported by National Science Foundation Grant 15948 to S.S. and National Institutes of Health Grant GM 32099 which supports the mariculture facility at the Marine Biological Laboratory. I wish to thank $R$. Woolley and L. Katz for their technical assistance, T. Capo and S. Perritt for raising the animals in this study, and $A$. Krawetz for typing the manuscript. I also thank Drs. D. Goldberg, V. Castellucci, and I. Kupfermann for their comments on earlier drafts of the manuscript.
}

which are later eliminated or retracted. For example, some identified neurons in several invertebrates extend axons into inappropriate regions during development which are eliminated after the cells contact their appropriate targets (Goodman and Spitzer, 1979; Kuwada, 1982; Wallace, 1984). The final organization of both peripheral and central connections of many neurons in the central and peripheral nervous systems of vertebrates occurs after extensive elimination of some processes and connections formed earlier in development (Devor and Schneider, 1975; Brown et al., 1976; Crepel et al., 1976; LeVay et al., 1980; Lichtman and Purves, 1980; Mariani and Changeaux, 1981; Reh and Constantine-Paton, 1984; Shatz and Kirkwood, 1984). These studies suggest that a number of mechanisms may be utilized by neurons in remodeling their neuritic arbor-limitations on the processes extended by particular neurons, the distribution of trophic or target-derived factors affecting neurite extension, competition among invading fibers for a limited number of sites within the target, and appropriate functional activity for stabilizing some connections and the elimination of others. Little is known, however, on how these various factors influence neuritic pruning or the intracellular mechanisms mediating the selective elimination or retraction of certain structures.

To explore these mechanisms, I have developed an in vitro system in which neurite outgrowth and synapse formation at different axon branches of a single identified Aplysia neuron, the metacerebral cell (MCC), can be examined under controlled conditions. This giant serotonergic neuron of the marine mollusc Aplysia californica has been studied extensively in terms of transmitter biosynthesis and transport (Eisenstadt et al., 1973; Weinreich et al., 1973; Goldman et al., 1976; Ambron et al., 1980), synaptic connections and morphology (Gerschenteld and Paupardin-Tritsch, 1974; Weiss and Kupfermann, 1976; Weiss et al., 1978; Schwartz and Shkolnick, 1981), and role in behavior (Susswein et al., 1976; Rosen et al., 1983). The axon of the MCC bifurcates within the cerebral ganglion. The larger axon branch is in the cerebral-buccal connective (CBC); it innervates several buccal ganglion neurons and, via branches in peripheral nerves of the buccal ganglion, innervates buccal muscle. The smaller diameter branch is in the posterior lip nerve (PLN) which terminates in lip musculature.

Using methods previously developed for Aplysia neurons in culture (Schacher and Proshansky, 1983), the MCC can be isolated with its bifurcate axon and can be maintained in culture without or with buccal neuron targets. I report here that the MCC re-establishes appropriate chemical connections with buccal neuron targets in greater than $90 \%$ of the cultures when the targets are contacted by neurites emerging from the $\mathrm{CBC}$ branch but forms connections in only $20 \%$ of the cultures when the same buccal targets are contacted by neurites emerging from the PLN branch. The two branches also show differential neurite outgrowth in the presence of targets. Growth from the PLN branch is markedly reduced only when neurites 
from the $\mathrm{CBC}$ branch contact and form chemical connections with the buccal neuron targets, compared to PLN growth in the absence of targets. Growth from the CBC branch is not affected by the presence of a target at either branch.

\section{Materials and Methods}

Tissue culture procedures and electrophysiology. The methods used for isolating Aplysia neurons, maintaining them in culture, and detecting chemical connections have becn described (Camardo ct al., 1983; Schacher and Proshansky, 1983). Buccal ganglia and cerebral ganglia from 2- to 5-gm animals raised from fertilization at the Marine Biological Laboratory at Woods Hole, Massachusetts (Kriegstein et al., 1974; Kriegstein, 1977) were bathed in proteolytic enzyme, pinned with glass microelectrodes in a Sylgaard (Dow Corning Corp., Midland, MI)-coated dish, and desheathed. The MCC, located on the dorsal surface of the ganglion near the midline, was removed by touching the cell body with a fine-tipped microelectrode and slowly pulling it in a direction opposite from the exiting MCC branches (Fig. 1). Following the transfer of the MCC to a culture dish containing a 1:1 mixture of the L15 (Flow Laboratories, Inc., McLean, VA) and Aplysia hemolymph, the two branches were gently teased apart and separated so that their axon stumps were well separated from each other. Identified buccal cells B1 and B2 on the caudal surface of the buccal ganglion (Gardner, 1971) were isolated in the same manner as the MCC. The cells (there are two bilaterally symmetrical pairs in each buccal ganglion) were identified on the basis of size, position and presence of an axon in the esophageal nerve. The identity of these cells was further verified by the presence of small cardioactive peptides $\left(\mathrm{SCP}_{\mathrm{A}}\right.$ and $\mathrm{SCP}_{\mathrm{B}}$ ) in single cells grown in culture (P. Lloyd, $\mathrm{S}$. Schacher, $\mathrm{K}, \mathrm{R}$. Weiss, and I. Kupfermann, manuscripl in preparation). The buccal neurons were transferred to the culture dish containing the MCC and positioned near the stump of the CBC branch or the PLN branch. The cultures were maintained at room temperature for the first $24 \mathrm{hr}$ and then were transferred to an incubator at $18^{\circ} \mathrm{C}$. The medium was changed every 2 days with the same L15-hemolymph mixture used at the initial plating

After 5 to 6 days the presence of chemical connections between the cells was monitored by standard intracellular recording methods. All intracellular recording was done with a single-barreled electrode of 10 to 15 megohms filled with $2.5 \mathrm{M} \mathrm{KCl}$. Sufficient current was injected into the MCC to produce trains of action potentials ( 3 to $15 \mathrm{~Hz}$ ) lasting 1 to $5 \mathrm{sec}$. To be scored as a chemical connection, responses in the buccal cells had to meet three criteria that are characteristic for this connection in vivo (Gerschenfeld and Paupar din-Tritsch, 1974; Weiss and Kupfermann, 1976). First, the latency of the response had to be less than $2 \mathrm{sec}$ from the onset of MCC action potentials. Second, the depolarization had to be sustained with an amplitude of $1 \mathrm{mV}$. Third, constant depolarizing or hyperpolarizing pulses in both cells indicated no evidence of electrotonic connections.

Measurements of neurite sprouting. All measurements of sprouting were made on the neurites extending from the stumps of the PLN and CBC branches. The extent of sprouting from a given branch was determined qualitatively by measuring the surface area of the culture dish encompassing the neurites emerging from that branch. The growth from each stump of an MCC was photographed on a Leitz inverted microscope and enlarged to equivalent magnifications. A circumferential line interconnecting the tips of the longest neurites was drawn to demarcate the area covered by growth from each stump. Measurements of growth from a stump in the presence of a buccal neuron target were made in two ways. In some cases $(N=6)$ growth was determined as described above, following intracellular injection of Lucifer Yellow (Stewart, 1981) into the MCC soma. In other cases $(N=$ 14) it was possible to distinguish MCC neurites from those emerging from the buccal neuron, permitting growth measurements as described above for cells without targets.

\section{Results}

MCC branches can be distinguished by axon diameter. Studies of MCC in vivo have shown that the axon diameter of the CBC branch is larger than that of the PLN branch. Isolated MCCs maintained in culture retain this difference in axon diameter (Fig. 2). The ratio (CBC:PLN) for axon diameter was $1.5 \pm 0.2(N=20)$. The identity of the branches was verified in early experiments by cutting the CBC shorter than the PLN before cell isolation $(N=5)$. The MCCs isolated under these conditions have a short large-diameter branch (CBC) and a long small-diameter branch (PLN).

Neurite outgrowth from the MCC branches. Neurite outgrowth begins from the swollen stumps of the two branches within $1 \mathrm{hr}$ after plating. By 5 days, the branches have elaborated an extensive network of neurites forming a nearly circular mat around the original PLN and CBC stumps (Fig. 2A). As with other Aplysia neurons in culture (Camardo et al., 1983; Schacher and Proshansky, 1983), the MCCs extend processes almost exclusively from their axon stumps (Fig. 2). The buccal neurons, placed adjacent to the branch stumps (Fig. 2, B and C), elaborate processes as well. Within $12 \mathrm{hr}$, neurites emerging from the MCC branches have contacted the buccal cell (either cell body, axon, or neurites). Because the MCC is isolated with branches that are long $(0.3$ to $1 \mathrm{~mm})$ and well separated, the buccal cells are contacted exclusively by neurites emerging from one branch (Fig. 2, B and C). This permits an examination of the formation of chemical connections and the extent of neurite outgrowth at each branch.

MCC branches show differential capacities in forming chemical connections with buccal neuron targets. The MCCs form unidirectional chemical synaptic connections with the buccal neurons within 5 days after plating (Fig. 3). A burst of action potentials in the buccal neuron has no effect on the MCC (Fig. $3 A$ ). The CBC branch is significantly more effective in forming a chemical connection than the PLN branch. When contacted by neurites emerging from the $\mathrm{CBC}$ branch (Fig. $2 B$ ), MCC-buccal neuron connections are present in more than $90 \%$ of the cultures (19 of 21). A burst of action potentials in the MCC ( 3 to $10 \mathrm{~Hz}$ for 1 to $3 \mathrm{sec}$ ) elicits a long-lasting depolarization (10 to $60 \mathrm{sec}$ ) in the buccal neuron which typically (15 of 19) is sufficient to produce a prolonged burst of action potentials in the buccal neurons (Fig. $3 A$ ). In contrast, buccal neurons contacted by neurites emerging from the PLN branch (Fig. $2 \mathrm{C}$ ) fail to show a chemical connection (Fig. 3C) in $80 \%$ of the cultures (20 of 25). In addition, the chemical connection present in $20 \%$ of the cultures ( 5 of 25 ) is weaker (Fig. $3 B$ ) than that seen at the MCC-buccal neuron connection via the CBC branch (Fig. $3 A$ ).

Neurite outgrowth from PLN is reduced selectively when target is placed near $C B C$. Under all conditions, neurite outgrowth from the CBC branch is greater than growth from the PLN (Figs. 4 to 8 ). Although there is considerable intercell variability, a comparison of the growth from the ditterent branches for any given MCC under different conditions indicates more extensive growth from the CBC (growth ratios of 1.2 to 4.5 ). In the absence of buccal neuron targets, the average CBC versus PLN growth ratio is 1.6 (Fig. 7A, left bar). This ratio is not significantly affected by the presence of a buccal cell at the PLN branch (Fig. 7A, right bar). However, the presence of a buccal cell at the CBC branch significantly increases the growth ratio to a value of 3.1 (Fig. $7 A$, middle bar)
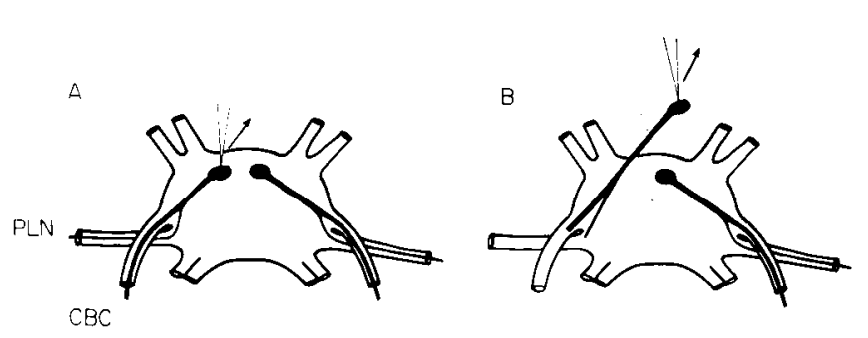

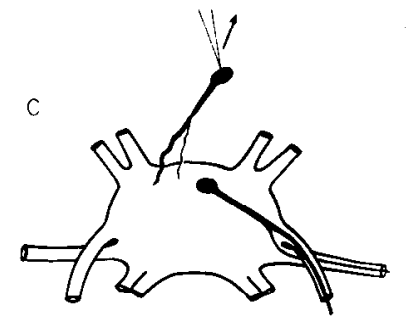

Figure 1. Isolation of MCC from cerebral ganglion. $A$, Following the desheathing of the ganglion, a microelectrode touches the cell body. $B$, Moving the electrode in the direction of the arrow, the cell is slowly displaced from the ganglion. $C$, The cell is displaced completely from the ganglion. 
Figure 2. MCC in culture after 5 days under various conditions. $A$, MCC from a 5-gm animal. The two branches are indicated. Note that all neuritic growth from the cell has emerged from the two branch stumps (arrowheads). B, MCC with buccal neuron $(B N)$ target positioned near the stump of the CBC branch. Note that the buccal cell is contacted by neurites emerging from the CBC stump only. Both cells were isolated from a 2-gm animal. $C$ MCC with buccal neuron target positioned near the stump of the PLN branch. Both cells were isolated from a 2-gm animal. Bars, $100 \mu \mathrm{m}$.
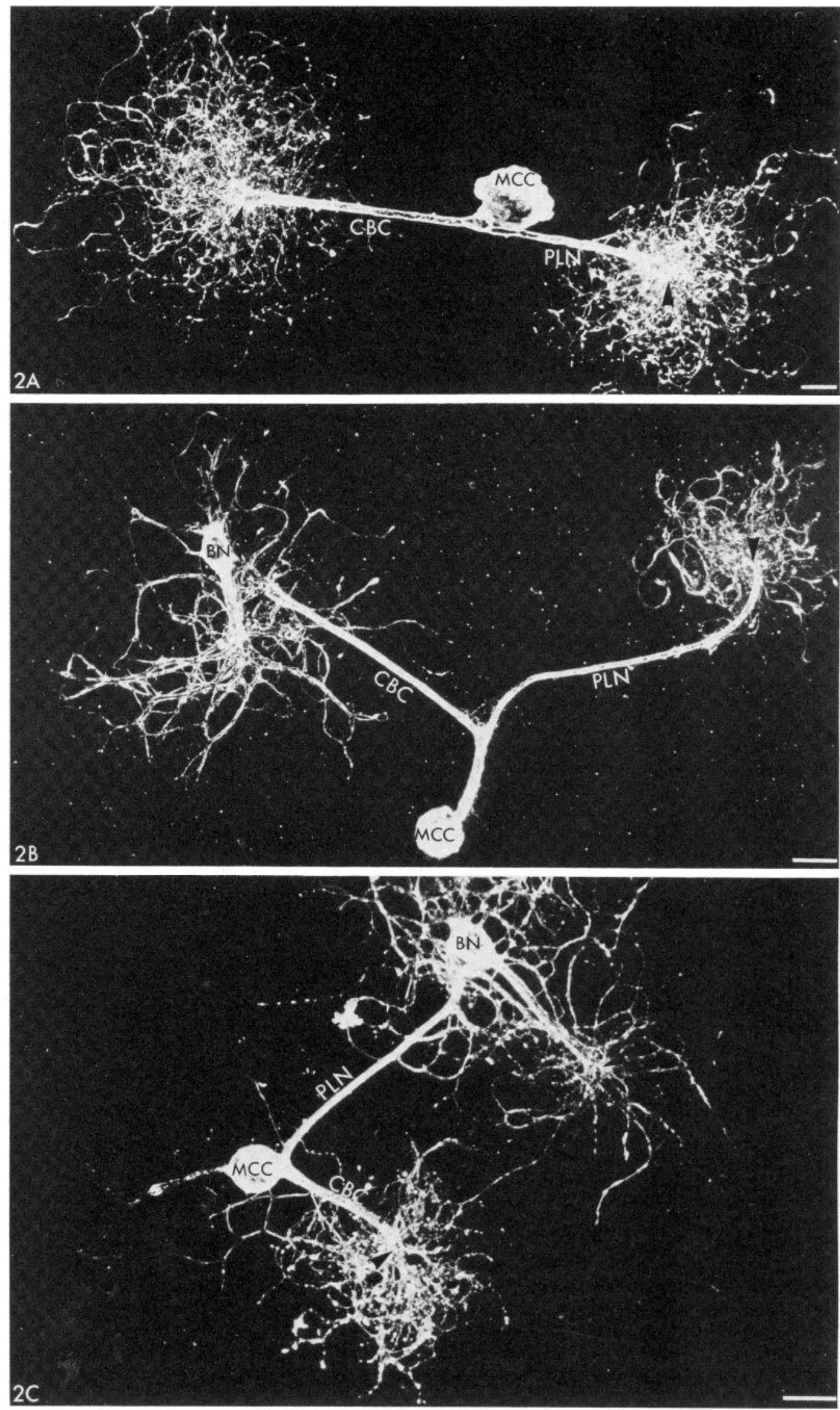

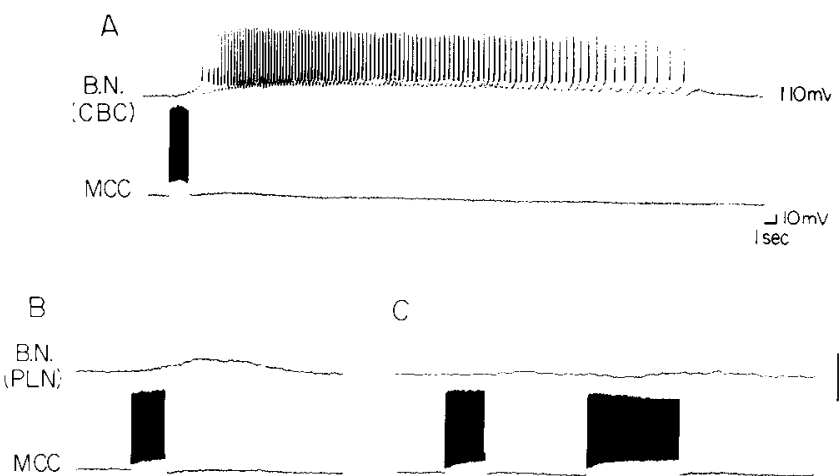

C

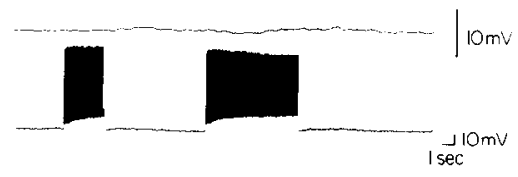

Figure 3. Chemical connections between MCC and buccal neurons after 5 days in culture. $A$, MCC-buccal neuron (B.N.) connection via the CBC branch. A brief burst of action potentials in the MCC produced a long-lasting depolarization in the buccal neuron resulting in the repetitive firing of action potentials. This type of connection was observed in 15 of 19 cultures in which a chemical connection via the CBC branch was present. $B$ and $C$, MCC-buccal neuron connection via the PLN branch. $B$, In 5 of 25 cultures, a burst of action potentials in the MCC produced a relatively weak depolarization in the buccal cell. $\mathrm{C}$, In the other $80 \%$ of the cultures, high frequency stimulation of the MCC produced little or no effect in the buccal cells.

To determine the factors contributing to the CBC versus PLN growth ratios, we examined the effects of target position on the overall growth from the two branches after 5 days. The results shown in Figure $7 B$ indicate that neurite outgrowth from the CBC branch is not significantly affected by the presence of a target at either branch (Fig. 7B, open bars). Neurite outgrowth from the PLN (Fig. 7B, hatched bars) is not significantly affected by the presence of a target at the PLN branch, but is reduced significantly when the target is placed by the $\mathrm{CBC}$ branch (Fig. $7 B$, middle and right bars). The reduction in PLN growth occurs without any significant change in the growth from the CBC branch.

Rate of neurite outgrowth from the PLN is selectively reduced when a buccal neuron is placed by the CBC branch. We next examined the effect of target position on the rate of neurite outgrowth to determine whether the decrease in growth from the PLN occurs immediately after plating or at some critical point during the 5-day culture period. In the absence of any targets, the rate of neuritic growth from the CBC and PLN is constant during the first 5 days (Fig. 8, $A$ and $B$, solid line). The rate of neuritic growth from the $\mathrm{CBC}$ in the presence of a target at the PLN shows a similar constant growth rate (Fig. $8 A$, dashed line). In contrast, the growth rate for the PLN in the presence of a CBC target is markedly reduced on the third day. The reduction in growth rate thus occurs between the first and third day, since the growth rate of the PLN on day 1 is not significantly affected by the presence of a target at the CBC (Fig. $8 B$, dashed line).

\section{Discussion}

The results reported here indicate that two major branches of a single neuron have different capacities in forming connections with the same target neurons. The CBC branch of the MCC, which normally innervates ncurons in the buccal ganglion, is more cffective in re-establishing chemical connections with $\mathrm{B} 1$ and $\mathrm{B} 2$ than is the PLN branch.

There are several possible explanations for these results. Because neurite outgrowth from the CBC branch is greater than that from the PLN, CBC neurites may form more contacts with the buccal neuron targets than do PLN neurites. Besides this increase in growth, each CBC neurite may contain more "release sites" than do those emerging from the PLN, and each release site on the CBC neurites may be more effective in transmitter release than those on the PLN. If a critical number of contacts is needed to permit the detection of a chemical connection with the intracellular recording methods de- scribed here, then MCC-buccal neuron connections via the CBC branch would be more effective than those connections formed via the PLN branch.

Although there is no direct evidence as yet to rule out thesc growth-related factors, the ability of the CBC branches to form strong connections with buccal neurons despite the variability in CBC outgrowth (Figs. 7 and 8) suggests that neurite outgrowth per se is not a major factor affecting the strength of MCC-buccal neuron contacts. Thus, $\mathrm{CBC}$ branches which show relatively poor neurite outgrowth (compared to other $\mathrm{CBC}$ branches and some PLN branches) form chemical connections as well as cells with CBC branches that have extensive growth. Similarly, cells with PLN branches that have extensive growth are not more likely to form connections than cells with less growth from either the PLN or the $\mathrm{CBC}$. Clearly, however, a closer examination of the morphology of MCC-buccal neuron contacts for each MCC branch is needed to rule out the contributions of growth-related and structural factors in the formation and strength of MCC-buccal neuron connections.

The ability of the CBC branch to form connections with buccal ganglion targets more effectively than does the PLN branch may result from some branch-specific cell interaction. The results concerning neurite outgrowth from the two branches - the decrease in growth from the PLN branch only when the CBC branch contacts and forms synaptic connections with the buccal neuron targets, as opposed to the unaltered neurite outgrowth from the CBC when the same targets are contacted by the PLN-is consistent with the idea that branch-specific cell interactions may be involved (see below). Cell-cell interactions, either "recognition" factors on the cell surface or releasable factors from pre- and postsynaptic cells (Sperry, 1963 Christian et al., 1978; Jessell et al., 1979; Gottlieb and Glaser, 1980; Salpeter et al., 1982; Edelman, 1983; Sanes et al., 1984), have been suggested as mechanisms by which neurons form specific connections. If such a mechanism is working here, then the results suggest that the cellular machinery underlying this interaction is selectively distributed over the two MCC branches. The segregation of functionally important macromolecules within mature cells, especially neurons, is well established. A similar segregation of developmentally relevant molecules may be crucial in allowing neurons with synaptic targets spatially distant from one another to form appropriate connections. A critical test of this hypothesis regarding synapse formation is to examine synapse formation by the MCC branches when the CBC and PLN branches are presented with the peripheral targets normally innervated by the PLN branch.

In the absence of targets, neurite outgrowth from the CBC is greater than growth from the PLN (ratio of 1.6). Since the diameter of the CBC branch is greater than that of the PLN, this difference in growth may involve the different capacity of the two branches to transport growth-related materials from the cell body (Ochs, 1982). Consistent with this notion is the observation that the ratio of fast axonal transport of certain materials in the CBC versus the PLN in the adult MCC is 1.7 to 1.8 (Aletta and Goldberg, 1982, 1984).

Neurite outgrowth from the two MCC branches can be affected selectively since MCC-target contact via the $\mathrm{CBC}$ results in a decrease in neurite outgrowth from the PLN while not changing growth from the CBC. Regeneration from the central and peripheral axons of dorsal root ganglion cells can be altered selectively under certain conditions and is thought to be associated with a differential change in slow axonal transport of growth-related materials (Oblinger and Lasek, 1984).

The selective reduction of neurite outgrowth capacity from the PLN branch may result from a change in the partitioning between the two branches of growth-related material moving by axoplasmic transport. For example, recent in vivo studies showed that, shortly after one branch of the MCC is detached from its synapses, transmitter storage vesicles are routed away from the damaged branch into the other branch despite the ability of the injured branch to transport its normal complement of vesicles (Aletta and Goldberg, $1982,1984)$. Perhaps a branch with synaptic endings is a preferred 
Figure 4. Lucifer Yellow-filled MCC branches contacting buccal neuron targets $(B N)$ facilitate measurement of growth from the $\mathrm{CBC}$ or PLN branches. $A, C B C$ branch contacts buccal neuron. $B$, PLN branch contacts buccal neuron. The arrowheads point to branch stumps. Bars, $100 \mu \mathrm{m}$.

Figure 5. Neurite outgrowth from two MCC branches with buccal neuron target at $\mathrm{CBC}$ branch. $A$, Growth from the $\mathrm{CBC}$ branch. The cell body of the buccal neuron is out of the field of view (lower right). $B$, Growth from the PLN branch of the same cell. Note the significant difference in the growth of the two branches. The arrowheads point to branch stumps. Bars, $100 \mu \mathrm{m}$.

Figure 6. Neurite outgrowth from two MCC branches with buccal neuron target $(B N)$ near PLN stump. A, Neurite outgrowth from the CBC branch is extensive and is not reduced as a result of target contact via the PLN. B, Neurite outgrowth from the PLN branch of the same cell. The arrowheads point to branch stumps. Bars, $100 \mu \mathrm{m}$.
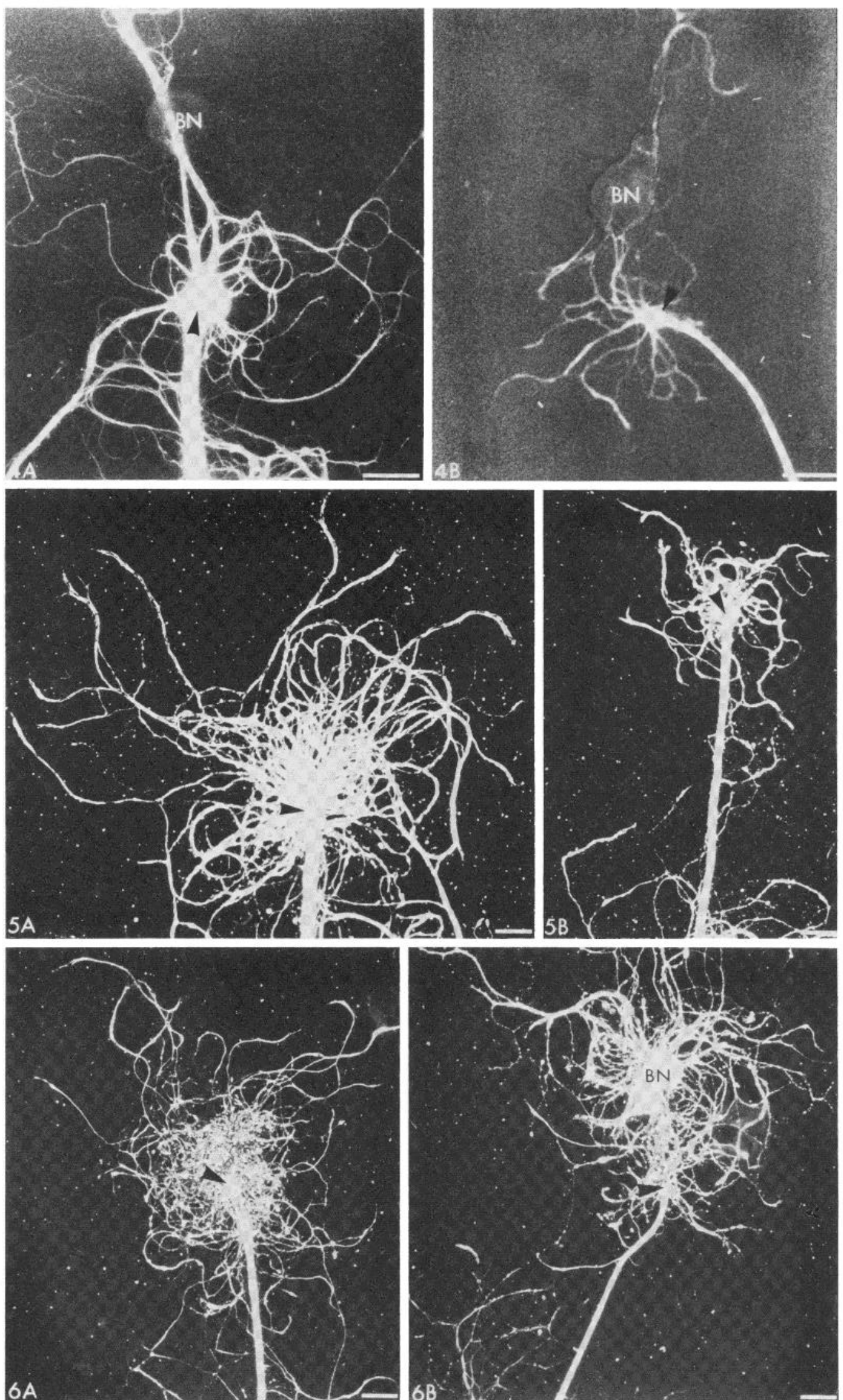
A

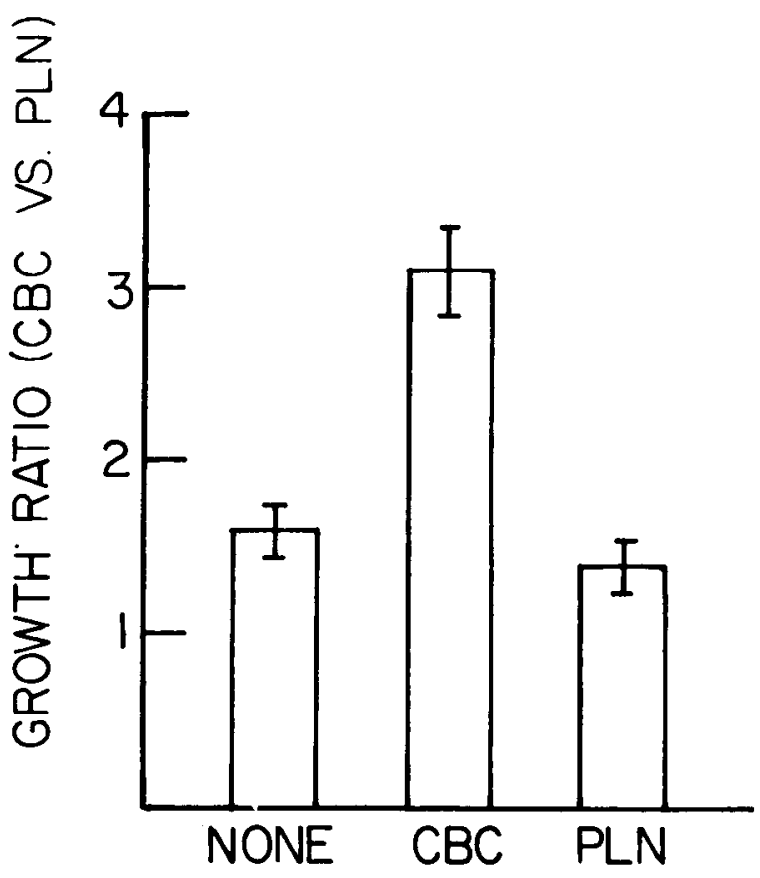

$\mathrm{B}$

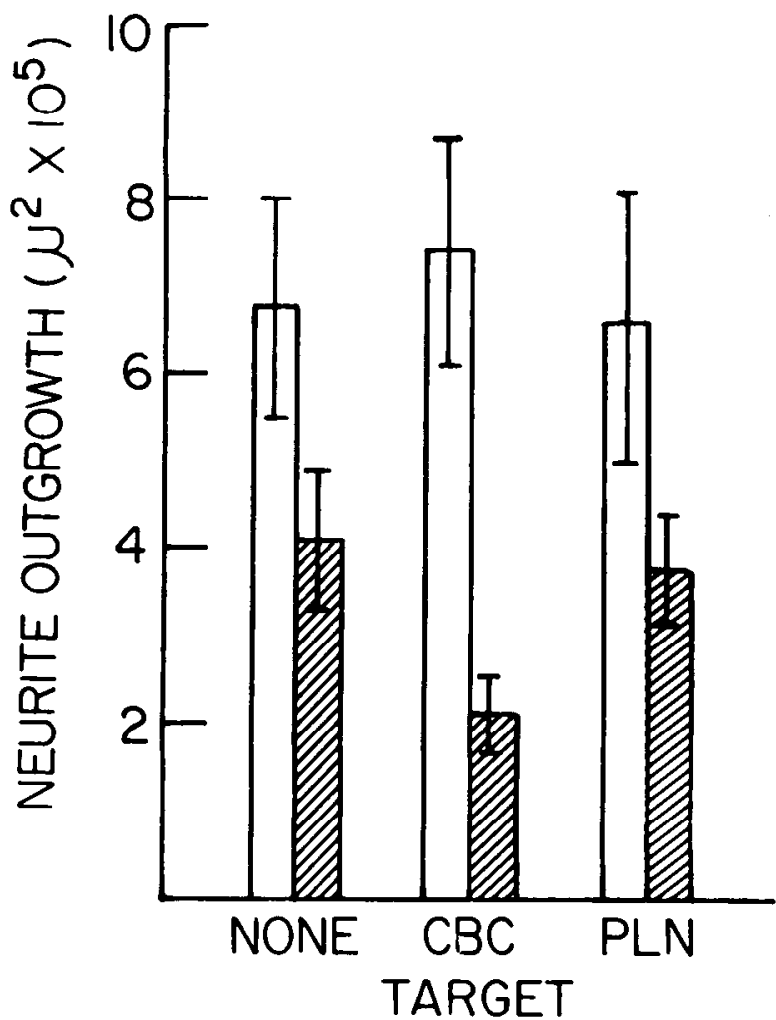

Figure 7. Neurite outgrowth from MCC branches with or without targets after 5 days in culture. $A$, Ratio of neurite outgrowth from MCC branches in the absence and presence of buccal neuron targets. Under all conditions, CBC growth is greater than PLN growth. The presence of a target at the $\mathrm{CBC}$ branch results in a significant increase in the growth ratio (one-way analysis of variance, $\rho<0.01$; and two-tailed $t$ test between groups, $\rho<$ $0.01)$ compared to the growth ratio in the absence of a target or when a target is placed by the PLN branch. The height of each bar is the mean \pm SE of measurements from 10 cells. $B$, Neurite growth from CBC and PLN branches. The extent of neurite outgrowth from the CBC branch (open bars) is not significantly affected by the presence of a target at either branch (one- route not only for transmitter vesicles but also for growth-related materials. If delivery of materials by axonal transport remains the same, however, then their use in the PLN axon or neurites must change selectively. Further studies should allow us to distinguish between these possibilities.

That the formation of contacts with the buccal neuron targets via the CBC may be a critical signal in the reduction of growth from the PLN is supported by the finding that the change in PLN growth occurs only after 1 day in culture, long after CBC neurites have contacted the target (more than $12 \mathrm{hr}$ ), and coincident with the first detection of chemical connection with the buccal cell (48 hr; data not shown). In addition, removal of connections via the $\mathrm{CBC}$ branch, by cutting the $\mathrm{CBC}$ axon in culture, results in a rapid restoration (within $24 \mathrm{hr}$ ) of ncuritc outgrowth from the PLN (S. Schacher and $P$. Edelstein, manuscript in preparation). Thus, information concerning the nature and extent of synaptic interactions can be rapidly transferred to the cell, resulting in appropriate alterations in growth. The rapid retrograde transport of information regarding local environmental conditions, such as the presence of nerve growth factor (Hendry et al., 1974; Thoenen and Barde, 1980; Richardson and Riopelle, 1984) or target-associated macromolecules (Hendry and Hill, 1980), by a specific branch of a neuron may allow the cell to direct its metabolic activities selectively to that branch. Clearly, the selective changes that may occur at these different branches are difficult to examine in vivo. The in vitro system described here may provide a means to study how a neuron regulates growth and
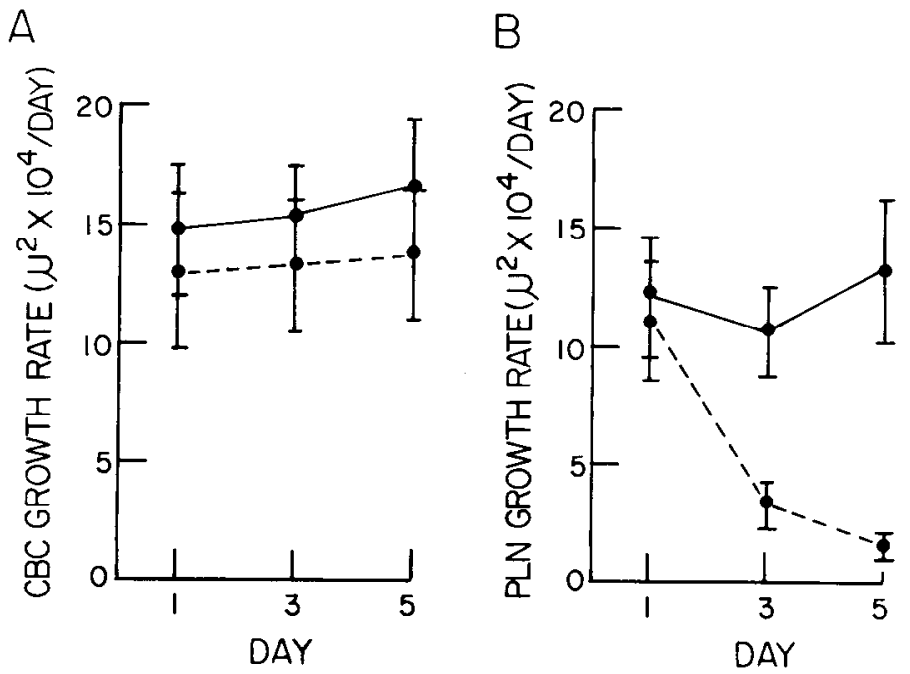

Figure 8. Rate of neurite outgrowth from $\mathrm{CBC}$ and PLN branches. A, Rate of neurite outgrowth from $\mathrm{CBC}$ branch in the absence of a target (solid line) and when a target is placed by the PLN branch (dashed line). Growth rate was measured as the per day change in the surface area of the culture dish circumscribing the neurites extending from the stumps of the PLN and CBC branches. Measurements were made on days 1, 3, and 5. A two-way analysis of variance indicates that the growth rate from the CBC is constant over the 5 -day perind and is not affected by the presence of a target at the PLN branch. Each point is the mean $\pm \mathrm{SE}$ of measurements from eight cells. $B$, Rate of neurite outgrowth from PLN branch in the absence of a target (solid line) and when a target is placed by the CBC branch (dashed line). A twoway analysis of variance indicates that neurite outgrowth from the PLN in the absence of a target is constant and decreases significantly $(\rho<0.01)$ when the target is placed by the CBC branch. A two-tailed $t$ test between groups indicates that growth rate is decreased on days 3 and $5(p<0.01)$ but is not different after 1 day in culture. Each point is the mean \pm SE of measurements from eight cells.

way analysis of variance, $p>0.2$ ). Ne.urite outgrowth from PLN branch (hatched bars) decreases significantly when the target is placed by the CBC branch (one-way analysis of variance, $p<0.02$; and two-tailed $t$ test between groups, $p<0.01$ ). The measurements wore made on the same cell populations described in Figure $7 \mathrm{~A}$. 
synapse formation at different branches and the nature of the cellcell interactions that may signal selective down regulation of neurite growth in some branches.

\section{References}

Aletta, J. M., and D. J. Goldberg (1982) Rapid and precise drawn regulation of fast axonal transport of transmitter vesicles in an identified neuron. Science 218: 913-916.

Aletta, J. M., and D. J. Goldberg (1984) Routing of transmitter and other changes in fast axonal transport after transection of one branch of the bifurcate axon of an identified neuron. J. Neurosci. 4: 1800-1808.

Ambron, R. T., J. E. Goldman, L. J. Shkolnick, and J. H. Schwartz (1980) Synthesis and axonal transport of membrane glycoproteins in an identified serotonergic neuron of Aplysia. J. Neurophysiol. 43: 929-944.

Barker, D. L., R. G. Wong, and S. B. Kater (1982) Separate factors produced by the CNS of the snail Helisoma stimulate neurite outgrowth and choline metabolism in cultured neurons. J. Neurosci. Res. 8: 419-432.

Bentley, D. and M. Caudy (1983) Navigational substrates for peripheral pioneer growth cones: Limb-axis polarity cues, limb-segment boundaries and guidepost neurons. Cold Spring Harbor Symp. Quant. Biol. 48: 573585.

Brown, M. C., J. K. S. Jansen, and D. Van Essen (1976) Polyneuronal innervation of skeletal muscle in new-born rats and its elimination during maturation. J. Physiol. (Lond.) 261: 387-422.

Camardo, J., E. Proshansky, and S. Schacher (1983) Identified Aplysia neurons form specific chemical synapses in culture. J. Neurosci. 3: 26142620.

Campenot, R. B. (198?) Development of sympathetic neurons in compartmentalized cultures. Dev. Biol. 93: 1-12.

Christian, C. N., M. P. Daniels, H. Sugiyama, Z. Vogel, L. Jacques, and P $G$. Nelson (1978) $\wedge$ factor from neurons increases the numbor of acctyl choline receptor aggregates on cultured muscle cells. Proc. Natl. Acad. Sci. U. S. A. 75: 4011-4015.

Crepel, F., J. Mariani, and N. Delhaye-Bouchard (1976) Evidence for a mulliple innervation of Purkinje cells by climbing fibers in the immature rat cerebellum. J. Neurobiol. 7: 567-578.

Devor, M., and G. Schneider (19/b) Neuroanatomical plasticity: The principle of conservation of total axonal arborization. In Aspects of Neural Plasticity, F. Vital-Durand and M. Jeannerod, eds., Vol. 43, pp. 191-201, INSERM, Paris.

Ebendal, T., L. Olsen, A. Sieger, and K. V. Hedlund (1980) Nerve growth factors in the rat iris. Nature $286: 25-28$.

Edelman, G. M. (1983) Cell adhesion molecules. Science 219: 450-457.

Eisenstadt, M., J. E. Goldman, E. R. Kandel, H. Koike, J. Koester, and J. H. Schwartz (1973) Intrasomatic injection of radioactive precursors for studying transmitter synthesis in identified neurons of Aplysia californica. Proc. Natl. Acad. Sci. U. S. A. 70: 3371-3375.

Gardner, D. (1971) Bilateral symmetry and interneuronal organization in the buccal ganglia of Aplysia. Science 173: $550-553$.

Gerschenfeld, H. M., and D. Paupardin-Tritsch (1974) On the transmitter function of 5-hydoxytryptamine at excitatory and inhibitory monosynaptic junctions. J. Physiol. (Lond.) 243: 457-481.

Goldman, J. E., K. S. Kim, and J. H. Schwartz (1976) Axonal transport of ${ }^{3} \mathrm{H}$ serotonin in an identified neuron of Aplysia californica. J. Cell Biol. 70. 304-308.

Goodman, C. S., and N. S. Spitzer (1979) Embryonic development of identified neurones: Differentiation from neuroblast to neuron. Nature 280 208-214.

Gottlieb, D. I., and L. Glaser (1980) Cellular recognition during neuronal development. Annu. Rev. Neurosci. 3: 303-318.

Hendry, 1. A., and C. E. Hill (1980) Retrograde axonal transport of target tissue-derived macromolecules. Nature 287: 647-649.

Hendry, I. A., K. Stoeckel, H. Thoenen, and L. L. Iversen (1974) The retrograde axonal transport of nerve growth factor. Brain Res. 68: 103-121.

Jessell, T. M., R. E. Siegel, and G. D. Fischbach (1979) Induction of acetylcholine receptors on cultured skeletal muscle by a factor extracted from brain and spinal cord. Proc. Natl. Acad. Sci. U. S. A. 76: 5397-5401.

Kriegstein, A. R. (1977) Development of the nervous system of Aplysia californica. Proc. Natl. Acad. Sci. U. S. A. 74: 375-378.

Kreigstein, A. R., V. Castellucci, and E. R. Kandel (1974) Metamorphosis of
Aplysia californica in laboratory culture. Proc. Natl. Acad. Sci. U. S. A. 71. 3654-3658.

Kuwada, J. Y. (1982) Primary axonal outgrowth of embryonic leech neurons. Neurosci. Res. Program Bull. 20: 877-881.

Letourneau, P. C. (1975) Cell-to-substrate adhesion and guidance of axonal elongation. Dev. Biol. 44: 92-101.

LeVay, S., T. N. Weisel, and D. H. Hubel (1980) The development of ocular dominance columns in normal and visually deprived monkeys. J. Comp. Neurol. 191: 1-51.

Levi-Montalcini, R. (1976) The nerve growth factor: Its role in growth, differentiation and function of the sympathetic adrenergic neuron. Prog. Brain Res. 45: 235-257.

Lichtman, J. W., and D. Purves (1980) The elimination of redundant preganglionic innervation to hamster sympathetic ganglion cells in early postnatal life. J. Physiol. (Lond.) 301: 213-228.

Mariani, J., and J. -P. Changeaux (1981) Ontogenesis of olivocerebellar relationships. I. Studies by intracellular recordings of the multiple innervation of Purkinje cells by climbing fibers in the developing rat cerebellum. $J$. Neurosci. 1: 696-702.

Oblinger, M. M., and R. J. Lasek (1984) A conditioning lesion of the peripheral axons of dorsal root ganglion cells accelerates regeneration of only their peripheral axons. J. Neurosci. 4: 1736-1744.

Ochs, S. (1982) Axoplasmic Transport and Its Relation to Other Nerve Functions, John Wiley \& Sons, Inc., Now York.

Raper, J. A., M. J. Bastiani, and C. S. Goodman (1983) Pathfinding by neuronal growth cones in grasshopper embryos. I. Divergent choices made by growth cones of sibling neurons. J. Neurosci. 3: 20-30.

Reh, T. A., and M. Constantine-Paton (1984) Retinal ganglion cell terminals change their projection sites during larval development of Rana pipiens. J. Neurosci. 4: 442-457.

Richardson, P. M., and R. J. Riopelle (1984) Uptake of nerve growth factor along peripheral and spinal axons of primary sensory neurons. J. Neurosci. 4: $1683-1689$.

Rosen, S. C., I. Kupfermann, R. S. Goldstein, and K. R. Weiss (1983) Lesion of a serotonergic modulatory neuron in Aplysia produces specific defect in feeding behavior. Brain Res. 260: 151-155.

Salpeter, M. M., S. Spanton, K. Holly, and T. R. Podiski (1982) Brain extract causes acetylcholine receptor redistribution which mimics some early events at developing neuromuscular junctions. J. Cell Biol. 93: 417-425.

Sanes, J. R., D. H. Feldman, J. M. Cheney, and J. C. Lawrence, Jr. (1984) Brain extract induces synaptic characteristics in the basal lamina of cultured myotubes. J. Neurosci. 4: 464-473.

Schacher, S., and E. Proshansky (1983) Neurite regeneration by Aplysia neurons in dissociated cell culture: Modulation by Aplysia hemolymph and the presence of the initial axonal segment. J. Neurosci. 3: 2403-2413.

Schwartz, J. H., and L. J. Shkolnick (1981) The giant serotonergic neuron of Aplysia. A multitargeted nerve cell. J. Neurosci. 1; 606-619.

Shatz, C. J., and P. A. Kirkwood (1984) Prenatal development of functional connections in the cat's retinogeniculate pathway. J. Neurosci. 4: 13781397.

Sperry, R. W. (1963) Chemoaffinity in the orderly growth of nerve fiber patterns and connections. Proc. Natl. Acad. Sci. U. S. A. 50: 703-710

Stewart, W. W. (1981) Lucifer dyes-Highly fluorescent dyes for biological tracing. Nature 292: 17-21.

Susswein, A. J., I. Kupfermann, and K. R. Weiss (1976) The stimulus control of biting in Aplysia. J. Comp. Physiol. 108: 75-96.

Thoenen, $H_{\text {., }}$ and U. A. Barde (1980) Physiology of nerve growth factor. Physiol. Rev. 60: 1284-1335.

Varon, S., and R. Bunge (1978) Trophic mechanism in the peripheral nervous system. Annu. Rev. Neurosci. 1: 327-361.

Wallace, B. G. (1984) Selective loss of neurites during differentiation of cells in the leech central nervous system. J. Comp. Neurol. 228: 149-153.

Weinreich, D., M. W. McCamon, R. E. McCaman, and J. E. Vaughn (1973) Chemical, enzymalic; and ullrastruclural characterization of 5-HT-containing neurons from the ganglia of Aplysia californica and Tritonia diomedia. J. Neurochem. 20: 969-976.

Weiss, K. K., and I. Kuptermann (1976) Homology of the giant serotonergic neurons (metacerebral cells) in Aplysia and pulmonate molluscs. Brain. Res. 117: 33-49.

Weiss, K. R., J. L. Cohen, and I. Kupfermann (1978) Modulatory control of buccal musculature by a serotonergic neuron (metacerebral cell) in Aplysia. J. Neurophysiol. 41: 181-208. 